\title{
Lidil
}

Revue de linguistique et de didactique des langues

$48 \mid 2013$

L'émotion et l'apprentissage des langues

\section{Entre émotions et contrôle de soi : un enjeu essentiel pour l'autonomie dans l'apprentissage des langues}

Stephen Scott Brewer

\section{OpenEdition}

Journals

Édition électronique

URL : http://journals.openedition.org/lidil/3331

DOI : 10.4000/lidil.3331

ISSN : 1960-6052

Éditeur

UGA Éditions/Université Grenoble Alpes

Édition imprimée

Date de publication : 1 novembre 2013

Pagination : 189-208

ISBN : 978-2-84310-260-8

ISSN : 1146-6480

Référence électronique

Stephen Scott Brewer, «Entre émotions et contrôle de soi : un enjeu essentiel pour l'autonomie dans l'apprentissage des langues », Lidil [En ligne], 48 | 2013, mis en ligne le 01 mai 2015, consulté le 10 décembre 2020. URL : http://journals.openedition.org/lidil/3331; DOl : https://doi.org/10.4000/lidil. 3331 


\title{
Entre émotions et contrôle de soi : un enjeu essentiel pour l'autonomie dans l'apprentissage des langues
}

\author{
Stephen Scott Brewer*
}

\begin{abstract}
RÉSUMÉ
Au moment où le métier de l'enseignant devient particulièrement difficile et les demandes sociétales dans de nombreux domaines, y compris les langues, se font de plus en plus pressantes, il devient nécessaire de réfléchir sur les enjeux liés à l'autorégulation des élèves et des étudiants face à leurs apprentissages. Le regard posé ici sur le rôle des émotions et du contrôle de soi dans l'acte d'apprendre est sociocognitif, mettant en relation le poids des facteurs internes de l'apprenant et des facteurs externes du contexte d'apprentissage. La notion de volonté est explorée, notamment dans sa dimension «instinctive». Concernant la pratique pédagogique, il est suggéré que les enseignants pourraient s'intéresser moins à la manière de motiver leurs élèves et davantage à la manière de les aider à se motiver eux-mêmes. Le passage d'une attitude à l'autre oblige à s'interroger sur les rapports entre émotions et contrôle de soi.
\end{abstract}

\section{ABSTRACT}

At a time when the teaching profession is becoming particularly difficult and society continues to express its rampant need for competence in numerous domains and disciplines, including foreign languages, it is becoming necessary to reflect on the stakes surrounding the issue of students' ability to self-regulate their learning. In this article the role of emotions and self-control in learning is studied from a sociocognitive perspective, which looks at the bidirectional influences of internal factors stemming from the learner and external factors stemming from the learning environment. The notion of will(power) is briefly examined in its "instinctive" dimension. Regarding teaching practice, it is suggested that teachers could be less interested in how to motivate their students and more interested in how to help them motivate themselves.

* Maitre de conférences, Université Paris-Est Créteil, Lidil 12 (EA 3958). 
The shift in attitude requires an inquiry into the relationship between emotions and self-control.

Il existe un proverbe rûmî ${ }^{1}$ qui dit : «Les hommes intelligents veulent le contrôle de soi; les enfants veulent des bonbons.» Ce vieil adage souligne l'importance de la capacité acquise des individus à contrôler ou à réguler la dynamique de leurs états internes, y compris de leurs émotions. L'apprentissage d'une langue non maternelle est une aventure qui affecte l'individu tout entier (Brown, 2007) et demande qu'il fasse montre d'un certain courage sur de nombreux plans : cognitif, affectif, comportemental, social, identitaire, etc. Personne ne saurait apprendre une langue sans motivation ${ }^{2}$ ni émotion positive (Oxford, 2012). À mesure que la compétence langagière se développe, les exigences d'apprentissage connaissent une augmentation exponentielle en termes de complexité cognitive et linguistique. Dans le même temps, le «retour sur investissement» en termes d'une amélioration de ses capacités communicatives devient de moins en moins perceptible. Tristement, la recherche signale bien trop souvent la baisse de la motivation des élèves une fois que l'effet de nouveauté associé aux premiers apprentissages d'une nouvelle langue s'estompe (Chambers, 1999; Little, Ridley \& Ushioda, 2002). Dans le domaine des langues, les apprenants de tout âge ont réellement besoin de leurs professeurs pour les aider à trouver du sens dans leurs apprentissages et à croire en leur capacité de réussir!

Or, si l'une des missions principales de l'enseignant doit être de créer des situations aptes à susciter la motivation et les émotions positives des élèves, comment cette mission relevant principalement de facteurs et de conditions externes s'articule-t-elle avec le développement interne

1. Djalāl ad-Dīn Muhammad Rūmī (1207-1273) est un mystique persan musulman dont les poèmes ont été traduits dans de nombreuses langues de par le monde.

2. Il existe une «extrême diversité» de théories de la motivation (Fenouillet, 2009, p. 9). Nous définissons celle-ci d'après les travaux de Ford (1992) qui conçoit la motivation comme un «construit intégré» (integrated construct, p. 78) composé de trois processus interreliés : les buts personnels (personal goals), les processus de stimulation émotionnelle (emotional arousal processes) et les croyances d'agentivité personnelle (personal agency beliefs). Ces processus servent respectivement à diriger, dynamiser et réguler le comportement ou l'action. Ainsi, pour nous, lorsqu'on parle de motivation, on parle nécessairement aussi d'émotion. 
de la capacité des élèves à gérer leur propre motivation? Si, d'une part, l'enseignant est là pour faire de l'apprentissage une source de plaisir et favoriser l'engagement motivationnel des élèves dans les tâches, comment fait-il, d'autre part, pour faire développer aux élèves la capacité non seulement à se motiver à se mettre au travail, mais également et peut-être surtout, à faire preuve de persévérance dans la durée ? Comment munir les élèves de ressources et de stratégies motivationnelles qui leur donnent la possibilité de maintenir leur effort et leur désir de travailler en dépit des inévitables distractions et difficultés? Comment leur apprendre à assurer le contrôle de leur motivation et de leur action en vue de la réalisation de buts d'apprentissage significatifs et souvent exigeants? Comment leur donner les moyens de savoir, vouloir et pouvoir différer le choix des «bonbons» (quand c'est nécessaire ou souhaitable) en faveur d'une hausse de leur capacité de contrôle dans l'effort et d'apprentissage autonome (Bouffard, 2011)?

Après avoir évoqué quelques enjeux importants liés au contexte de notre questionnement, nous proposons de définir les notions contenues dans le titre : émotion(s) et contrôle de soi. Ensuite, nous introduisons un concept connexe à celui de contrôle : la volonté (ou willpower, en anglais). Nous tenterons de montrer que, contrairement à ce que l'on pourrait croire, l'exercice de la volonté puise ses racines dans une caractéristique humaine foncièrement instinctive. À la fin de l'article, nous explicitons quelques «grandes lignes» de l'approche sociocognitive qui sert de cadre de référence à notre réflexion (Bandura, 2003). Nous concluons en rejoignant Puozzo Capron (2009) par rapport à la nécessité de concevoir un «nouvel enseignement/apprentissage des langues».

\section{Quelques enjeux contextuels}

Parvenir à la maitrise (relative) d'une langue non maternelle sans bénéficier d'une importante immersion linguistique à l'étranger - ce qui demeure le cas de la majorité des élèves et des étudiants - exige des années d'efforts soutenus. Le projet ministériel en France visant à rendre chaque élève capable de communiquer dans au moins deux langues vivantes à la fin de l'enseignement secondaire correspond à une réelle ambition sociale et éducative. Les couts associés à ce projet sont élevés ainsi que les attentes de résultats à tous les niveaux de la société française. Au niveau européen, le message est déployé depuis bientôt vingt ans (Commission européenne, 1995) selon lequel il faut poursuivre, en termes d'éducation et de formation, un seul et même 
objectif, à savoir la «prépar[ation] [d]es Européens à passer sans heurts à une société fondée sur l'acquisition des connaissances, où l'on ne cesse d'apprendre et d'enseigner tout au long de la vie, autrement dit, à une société cognitive» (Carré, 2005, p. IX), dont un élément fondateur est le plurilinguisme (CE, 1995, p. 10). Aussi semblerait-il pertinent de réfléchir aux manières de soutenir les initiatives autodirigées des élèves afin de favoriser l'exercice de leur contrôle personnel sur leurs apprentissages en langues et le développement de leur capacité à faire des choix utiles et à se sentir de plus en plus responsables de l'évolution de leurs compétences langagières au fur et à mesure qu'ils grandissent.

Cela étant, l'appropriation d'une nouvelle langue plus tard dans la vie pose tout un ensemble de défis, et ce, notamment sur le plan affectif et émotionnel. Horwitz (1999, p. XII) maintient que l'apprentissage d'une langue place le sujet apprenant de tout âge dans une position particulièrement vulnérable sur le plan psychologique et même quelque peu en porte-à-faux, à savoir celle qui consiste à avoir à communiquer quelque chose de significatif pour soi dans une langue que l'on ne maitrise pas suffisamment bien pour le faire. Selon Allwright et Bailey (1991), s'exprimer dans une langue qui n'est pas la sienne peut susciter des niveaux élevés de stress et d'anxiété, alors que voici trente ans que Guiora (1983) affirme avec force que l'apprentissage d'une nouvelle langue est «une proposition psychologique profondément déstabilisante» (p. 8). Enfin, le caractère «étranger» de la langue non maternelle peut déclencher des réactions affectives aussi bien positives que négatives en fonction des potentialités ouvertes par les valeurs culturelles associées à cette langue. Pour toutes ces raisons et bien d'autres encore, les apprenants en langues ont de quoi se sentir émotionnellement stimulés, parfois positivement, mais souvent le contraire (Horwitz, Horwitz \& Cope, 1986; Schumann, 1997; MacIntyre, 1995; Brewer, 2010).

Du point de vue développemental, les enjeux en matière d'autodirection prennent de l'ampleur au début de l'adolescence lorsque les élèves entrent au collège.

En passant d'un environnement scolaire personnalisé de pairs familiers à un environnement scolaire dépersonnalisé, $[\ldots]$ les jeunes adolescents perdent un certain sentiment de contrôle, ont moins confiance en euxmêmes, sont plus sensibles à l'évaluation sociale et souffrent d'une baisse de motivation personnelle. (Eccles \& Midgley, 1989, dans Bandura, 2003, p. 270-271) 
La recherche a montré que les croyances des collégiens en leur efficacité personnelle dans les domaines social et scolaire affectent leur bien-être émotionnel et leur développement (Bandura, 2003). L'adolescence correspond ainsi à un stade de développement où les jeunes vivent des expériences qui sont déterminantes par rapport à la façon dont celles-ci les préparent (ou non) à assumer le statut et les responsabilités du jeune adulte et au-delà. C'est une période critique où chaque jeune se forge une identité et prend des décisions importantes pour son avenir, notamment en lien avec les études, dont les langues font partie.

\section{Définitions conceptuelles}

L'objet de cette partie est de proposer des définitions pour les concepts clés qui sont au centre de cette contribution - émotion(s) et contrôle de soi - et de discuter brièvement leurs relations. Les émotions constituent un aspect essentiel de la vie mentale et sociale des individus, et leur fournissent un mécanisme très puissant pour réguler leurs comportements (Frijda, 1988; Zajonc, 1984). Comme l'affirme LeDoux (1996, p. 25), «des esprits sans émotions ne sont pas de véritables esprits. Ce sont des âmes prises dans la glace - des créatures froides, sans vie, privées de tout désir, de toute peur, douleur, souffrance, et de tout plaisir ${ }^{3} \gg$. À l'heure actuelle, une majorité de psychologues cognitifs estime que la cognition a besoin de l'émotion pour fonctionner correctement (Syssau, 2006, p. 57; Damasio, 1995). En effet, cette conception de l'émotion se démarque radicalement de la vision classique, dominante en Occident depuis l'Antiquité (Luminet, 2002), selon laquelle les émotions seraient une force perturbatrice, voire «désorganisatrice», empêchant l'homme de s'adapter efficacement à l'environnement (Janet, 1928, cité par Syssau, 2006, p. 16).

Dans une perspective sociocognitive, nous concevons les émotions comme n'étant créées ni par des processus purement intrapsychiques ni par l'environnement. Tout comme d'autres catégories de «représentations motivationnelles» (Bourgeois, 2011, p. 239), les émotions résultent de l'interaction entre facteurs internes, propres à l'individu, et

3. «Minds without emotions are not really minds at all. They are souls on ice-cold, lifeless creatures devoid of any desires, fears, sorrows, pain or pleasure.» 
facteurs externes, propres à la situation et au contexte. Cela dit, intrapsychiquement, nous considérons que les émotions sont des phénomènes neurophysiologiques qui «naissent», pour ainsi dire, au point de rencontre du corps et du mental, impliquant à la fois réactions corporelles et activités cognitives (en relation avec des évènements se produisant dans le monde). Les émotions constitueraient ainsi une forme de réaction (située) du corps en relation au mental, ou encore le reflet (situé) du mental dans le corps.

De nos jours, il n'est plus possible sur le plan scientifique d'opposer émotion et cognition ni d'«envisager une primauté temporelle de l'une sur l'autre. [...] Une séquence temporelle fixe qui débuterait soit par les réactions corporelles soit par l'évaluation [cognitive] n'a plus de raison d'être» (Syssau, 2006, p. 48). "Toutes nos opérations cognitives relèvent d'un ensemble fonctionnel extraordinairement complexe [...]. Le concept de "raison pure" est difficilement soutenable en ce qu'il isole le jeu continuel et réciproque de la relation entre pensée, ressenti émotionnel et comportement» (Trocmé-Fabre, 1999, p. 76). Or, si ces deux «grandes dimensions de l'esprit» ne sont pas séparables, si rationalité et affectivité sont, comme le suggère Trocmé-Fabre (1999), «les deux versants d'une même réalité» (p. 76), il n'est pas question de renoncer pour autant à leur distinction conceptuelle. Le défi qui nous interpelle est de déceler leur articulation, de comprendre leur collaboration (Syssau, 2006, p. 45), tout en soulignant l'impossibilité d'étudier l'une indépendamment de l'autre. On évoque à ce propos la complexité des liens entre le corps, la cognition, la conscience et l'émotion (ibid., p. 50).

Dans le cadre de la présente réflexion, nous privilégions l'examen du lien directionnel qui mène de la cognition et de la conscience vers l'émotion et le corps, non pas pour que celles-là «dominent» ou «contrôlent» ceux-ci, mais afin que la pensée et la capacité à prendre du recul par rapport à l'expérience puisse, à «leur façon », contribuer à une amélioration du bien-être psychique et émotionnel (voir Mayer \& Salovey, 1995 sur la notion d'intelligence émotionnelle). Puisque l'homme est «couplé à son environnement biologique par l'intermédiaire de la conscience» (Bateson, cité par Wittezaele, 2003, p. 75), il a la capacité à se mettre à distance de son expérience immédiate, y compris de son corps et de ses pensées propres. Ainsi, doté de la capacité de «savoir qu'il sait ${ }^{4} »$, l'homme a également la capacité de réfléchir à son

4. Ou, du moins, de la capacité de croire qu'il sait qu'il sait. 
propre vécu motivationnel. Selon Bandura (2003, p. 122), se motiver soi-même et adopter des conduites avec intention sont deux processus enracinés dans l'activité cognitive (Bandura, 2003, p. 122). «[L]a question de l'existence d'une évaluation dans le processus émotionnel ne semble plus être discutée. En effet, il y aurait dans toute expérience émotionnelle une évaluation cognitive de la situation.» (Musch \& Klauer, 2003, cités par Syssau, 2006, p. 43)

Quant à la notion de contrôle de soi, nous la concevons comme une sous-catégorie de la régulation de soi. Si certains chercheurs conçoivent l'autorégulation comme une activité que les individus adoptent à certains moments et non à d'autres sur la base, par exemple, de leur volonté propre (Zimmerman, 1990; Schunk, 1990), nous partons du principe que tout comportement humain est dans une certaine mesure «autorégulé» (à l'exception de certains réflexes ou activités autonomiques). Ainsi, pour nous, à l'instar de Ridley (1991), l'autorégulation serait considérée «comme une activité constante et continue, aussi naturelle et nécessaire que la respiration» (p. 32). En revanche, les notions de contrôle de soi, de maitrise de soi, de gestion de soi, ou encore d'autodirection relèvent de formes de la régulation du soi que nous considérons comme plus particulières. Ces nuances conceptuelles soulèvent la question des différences entre des aspects automatiques et contrôlés du fonctionnement humain, un champ de réflexion qu'il serait trop long de développer ici (voir Roeser \& Peck, 2009). Effectivement, selon Posner et Snyder (1975), la question de la conscience et du degré de contrôle conscient que les individus exercent sur leurs jugements, leurs décisions et leurs comportements est sans doute l'une des questions les plus importantes et fondamentales concernant l'existence humaine.

\section{La motivation extrinsèque requiert-elle plus de volonté?}

Un aspect plus spécifique encore de notre objet concerne la gestion de l'émotion sous forme de «volonté», appelée willpower, en anglais (McGonigal, 2012; Wolters, 1998). Ce concept a plus trait à la motivation d'apprendre extrinsèque qu'à la motivation d'apprendre intrinsèque (Deci \& Ryan, 1991). À condition d'en avoir le choix, nous supposons que la majorité des jeunes qui apprennent les langues à l'école ou à l'université optent à priori de les étudier en fonction du bénéfice qu'ils attendent de l'acquisition de cette compétence pratique, c'est-à-dire, en fonction d'un but dit «extrinsèque» (extérieur à l'activité d'apprendre 
une langue en elle-même). Par contraste, l'élève ou l'étudiant intrinsèquement motivé à apprendre une langue choisira de s'engager dans cet apprentissage pour le seul plaisir et la satisfaction que l'étude d'une langue suscite en soi. Même si nous n'en excluons pas la possibilité, nous partons du principe que relativement peu d'apprenants en langues non spécialistes ambitionnent de faire des langues leur matière de prédilection, mais voient plutôt en leur maitrise un avantage certain pour leur avenir.

La différence entre les deux types de motivation a des conséquences non négligeables sur la façon générale dont les élèves et étudiants diversement motivés auront tendance à réguler leurs apprentissages en langues, notamment du point de vue affectif. Si l'élève intrinsèquement motivé pour apprendre une langue a de fortes chances de s'adonner à ses études sans ressentir le besoin d'avoir à réguler ou à contrôler outre mesure son comportement (car il trouve ce qu'il fait à ce point plaisant et intéressant), il est bien plus probable que l'élève extrinsèquement motivé aura à «faire des efforts sur lui-même» afin d'atteindre des buts en langues qui comptent pour lui. Pour ce genre d'apprenant, la régulation de la motivation, et partant, des émotions, est plus susceptible de devenir un problème, peut-être non pas tant au moment de la décision de s'engager dans l'action que dans la perpétuation de celle-ci jusqu'à son terme. Dans le cas d'un apprentissage d'une langue, il semble souhaitable, voire nécessaire, de pouvoir (et savoir?) adopter ces deux façons complémentaires de réguler ses activités d'étude et ses expériences sociales.

La volonté est loin d'être un concept rationnel. Comme chacun de nous peut le constater par expérience personnelle, nous ne prenons pas toujours nos décisions en nous fondant sur des analyses rationnelles, loin s'en faut. Au contraire, le concept de volonté a précisément pour fonction d'essayer d'éclairer le passage complexe menant de l'intention à l'action (Cosnefroy, 2011, p. 39). La capacité humaine à mener une vie volontaire (volitional living) et à apprendre avec volonté (volitional learning) «rend possible non pas la liberté par rapport aux conditions extérieures, comme Frankl (1962) l'a dit, mais la liberté d'attitude envers ces conditions; non pas seulement l'adaptation aux environnements culturels, comme Maslow (1968) l'a dit, mais la capacité à transcender de tels environnements» (Roeser \& Peck, 2009, p. 119). Quant à la relation entre volonté et motivation, on pourrait prétendre que l'utilité d'avoir à faire preuve de volonté dans la vie est inversement proportionnelle à la nécessité d'être motivé. 


\section{L'instinct de volonté?}

L'instinct est cette partie de nous-mêmes (et d'autres espèces animales) où les tendances à agir sont innées, involontaires, impératives. Il peut ainsi paraitre quelque peu saugrenu de se demander si notre instinct (du côté de l'involontaire) nous équipe d'une capacité contraire de ce qu'il est lui-même (du côté du volontaire). Le devenir de cette capacité humaine à transcender non seulement des environnements mais aussi sa propre nature s'inscrit dans une tendance plus générale de la vie, dont la nature, selon Piaget, est «toujours de se dépasser» (Piaget, 1971, dans Deci \& Ryan, 1991, p. 239). Cette capacité au dépassement de soi que l'on observe dans la nature prend une forme toute particulière chez l'homme. Elle est étroitement liée au développement d'une partie spécifique du cerveau nommée le cortex préfrontal (Sapolsky, 2004). Au cours de millions d'années, cette zone du cerveau s'est développée et a commencé à assurer des fonctions de plus en plus complexes et à permettre aux êtres humains de mieux réguler leurs comportements. Selon Sapolsky (2004), la fonction principale du cortex préfrontal moderne est de fournir aux membres de notre espèce la capacité à faire ce qu'il appelle «le choix difficile» (the harder thing, en anglais). Cette capacité permet à l'individu de se convaincre de la nécessité de se lever quand il aimerait mieux rester couché, ou de se rappeler les raisons pour lesquelles il n'est pas souhaitable de grignoter entre les repas.

Or, selon McGonigal (2012), ces tendances en matière d' " autostart» («je me lève même quand je ne veux pas») et d'«auto-stop» («je ne grignote pas même quand j'en ai envie») ne seraient aucunement efficaces sans la capacité à se souvenir de ce que l'on désire à plus long terme. D'un point de vue neurologique, le cerveau est constitué de manière à soutenir ces trois capacités. Chose intéressante, cet aspect si intime de notre rapport à nous-mêmes puise ses racines dans notre socialité. Sous de très anciennes «pressions » ayant incité nos lointains ancêtres à être de meilleurs voisins, parents et compagnons, ils ont développé, et nous ont légué, ce qui peut être considéré comme une forme de proto-autocontrôle. C'est parce que nous vivons en communauté que nous avons appris à réguler nos appétits, nos tendances agressives et nos envies de sexe.

Le problème que nous rencontrons aujourd'hui est que, si le «modèle standard» semble fonctionner plutôt bien, force est de constater, grâce à l'appui d'études faites depuis les années 1980, qu'il devient urgent d'essayer d'améliorer les performances du modèle de base (voir 
Berglas \& Baumeister, 1993 et Baumeister, Heatherton \& Tice, 1994, sur le thème de la défaillance du contrôle de soi ou self-regulation failure). Pourquoi, si nous possédons la capacité à «faire le choix difficile» (Sapolsky, 2004), nous arrive-t-il souvent d'avoir envie de faire exactement le contraire?

Au cours de son développement, le cerveau humain n'a pas vraiment changé, mais plutôt a évolué. La différence sémantique n'est pas neutre. Au lieu de partir de zéro, l'évolution préfère ajouter de nouvelles couches sur celles qu'elle a déjà créées. Au fur et à mesure que nos ancêtres développaient de nouvelles compétences et que l'environnement rendait la maitrise de celles-ci de plus en plus pertinentes et utiles pour la survie de l'espèce, le cerveau primitif n'a pas été remplacé par un modèle tout neuf. Le système d'autocontrôle a été progressivement greffé sur le vieux système axé avant tout sur la gestion des pulsions et des appétits instinctifs. La conséquence en est que la tendance à agir par impulsion fait tout aussi partie intégrante de notre répertoire comportemental que la capacité à faire le choix difficile. Compte tenu des conduites que chacun de nous est capable d'adopter dans certaines circonstances lorsqu'il se trouve dans certains états physiques et/ou mentaux, il est tout à fait légitime de se demander si, en effet, chacun est bien le propriétaire d'un cortex préfrontal qui marche. Les luttes internes que nous livrons face à la tentation, à la procrastination et aux aléas de nos processus affectifs, cognitifs et motivationnels, ne sont pas tant le signe de nos faiblesses personnelles que la simple preuve que nous sommes bien membres de l'espèce humaine. S'il est toujours possible de ne pas céder à la tentation d'agir, cette possibilité ne garantit en rien qu'elle devienne réalité.

Imaginons, cependant, que nous nous trouvons face à un danger extrêmement grave. Pour nous permettre de réagir efficacement dans de pareilles circonstances, la nature n'a pas fait preuve de dilettantisme, au contraire. Quand nous vivons des épisodes de ce genre, le système nerveux central est équipé pour produire le comportement le plus impulsif possible. En rapport avec ce constat, de nombreux travaux de recherche ont bien montré ces dernières années (Damasio, 1995; Kitayama \& Howard, 1994) à quel point les émotions, telles que la peur ou le désir, jouent un rôle essentiel dans le bon fonctionnement de l'organisme. Sans peur, par exemple, nous ne saurions nous protéger de dangers imminents et futurs. Sans désir, il est vraisemblable que nous tomberions dans la dépression.

En ce qui concerne des types de dangers qui peuvent menacer notre santé et bien-être, McGonigal (2012) fait remarquer qu'il est abso- 
lument crucial de faire une nette distinction entre, pour prendre son exemple, un tigre à dents de sabre et une part de cheesecake. Tous deux ont, en quelque sorte, le potentiel de compromettre nos ambitions de vivre mieux et plus longtemps. Le tigre, pour des raisons évidentes, le cheesecake, parce qu'à force d'en consommer trop, il peut entrainer des troubles cardiaques. Or, dans l'un et l'autre cas, non seulement la nature de la menace n'est pas du tout la même, mais nous sommes dotés de la capacité à formuler des réactions fort différentes aux deux types de menace.

Dans le cas du tigre, la réaction commence par la perception de la menace externe, et engendre la réponse dite «lutte ou fuite» (en anglais, fight or flight). Celle-ci permet une gestion optimale de l'énergie et orchestre, en une fraction de seconde, une répartition complexe des ressources énergétiques que l'organisme a à sa disposition. Dans le cas du cheesecake, en revanche, la réaction commence avant tout par la perception d'un conflit interne. Le cheesecake, contrairement au tigre, n'est pas la vraie menace; l'«ennemi» est au-dedans de soi! Pour bien gérer ce genre de situation, l'être humain a hérité d'un autre genre de réponse : "prendre une pause pour réfléchir» (en anglais, pause and plan). Se sentir capable de marquer un temps d'arrêt au moment où l'envie du cheesecake s'empare de tout son être est un geste clé. Il permet de mettre le corps dans un état plus calme, tout en s'accordant un «espace de liberté» où il est possible d'avoir des pensées plus souples et réfléchies.

Enfin, si, comme cela a été montré par la recherche scientifique, la réaction «pause pour réfléchir» constitue une réponse tout aussi naturelle ou instinctive que la réaction «lutte ou fuite», pourquoi arrive-t-il, au quotidien, que nous ayons souvent l'impression qu'elle nous serve moins bien ou qu'elle fonctionne de manière moins instinctive? Selon McGonigal (2012), de nombreux facteurs affectent la «réserve de volonté» des individus, de ce qu'ils mangent à la qualité de l'air qu'ils respirent en passant par leurs propres états internes. Ainsi, la boucle est bouclée : comme cela est précisé dans la théorie sociocognitive, que nous sommes sur le point de présenter brièvement, les individus «sont à la fois les produits et les producteurs de la structure sociale, qui est, elle-même, vue comme à la fois un produit et une cause de l'action» (Carré, 2004, p. 38). 


\section{L'approche sociocognitive : un cadre théorique adapté à l'étude de la question}

Pour l'essentiel ${ }^{5}$, le cadre de référence dans lequel nous inscrivons notre réflexion est la théorie sociocognitive d'Albert Bandura (2003, 2009). Cette approche de l'étude du fonctionnement humain et de la motivation s'appuie sur un principe épistémologique fort pertinent : le déterminisme réciproque. Celui-ci propose d'étudier les phénomènes humains complexes, comme la régulation des états émotionnels, à travers l'interaction qui existe entre trois séries de facteurs : les comportements d'une personne, ses caractéristiques individuelles et l'environnement dans lequel elle évolue. C'est au carrefour de cette structure causale interdépendante qu'émerge et opère ce que Bandura appelle l'agentivité humaine (human agency).

"Être un "agent" signifie faire en sorte que les choses arrivent par son action propre et de manière intentionnelle» (Bandura, 2009, p. 17). L'agentivité s'enracine dans quatre capacités humaines fondamentales : 1) imaginer, anticiper et planifier l'avenir; 2) agir intentionnellement; 3) motiver et réguler sa propre activité; et 4) examiner réflexivement ses propres actions et pensées et leurs conséquences. Dans cette perspective, la capacité à pouvoir agir sur son émotivité par la réflexion intentionnelle constitue l'une des plus fortes ressources motivationnelles chez l'homme (Ridley, 1991). Chacun a potentiellement la possibilité d'apprendre à se percevoir et à se comprendre non seulement comme quelqu'un qui se contente d'être, mais également comme quelqu'un qui devient et choisit de participer activement à sa

5. Nous disons «pour l'essentiel» parce que, suivant Cosnefroy (2011), nous estimons que le modèle de l'apprentissage autorégulé, d'inspiration sociocognitive, proposé par Zimmerman (2000) a tendance à présenter le concept central de sa théorie fondatrice, l'auto-efficacité (self-efficacy), comme s'il suffisait à lui seul à rendre compte de la réussite du processus d'autorégulation. Comme Cosnefroy (2011) le suggère, «soucieux de privilégier une perspective agentique de l'apprenant, [ce modèle semble] mettre en avant les marques essentielles de cette agentivité [...] au risque de sous-estimer les contraintes environnementales et les spécificités disciplinaires des apprentissages. Y aurait-il dans le modèle de Zimmerman, et plus généralement dans la perspective sociocognitive, une difficulté à penser la dépendance du sujet par rapport à son environnement social?» (p. 48). Nous restons intellectuellement fidèle à la théorie de Bandura dans ses fondements épistémologiques, tout en refusant de suivre cette «pente naturelle» de la théorie (ibid., p. 48). 
propre évolution. Chacun est à la fois structure et fonction, «contenu» et «processus» (ibid., 1991, p. 31).

Sur le plan éducatif, l'exercice de l'agentivité soulève la question de savoir comment les élèves et étudiants apprennent à contribuer causalement, pour le meilleur comme pour le pire, à la réalisation effective de leurs propres apprentissages. Une perspective agentique de l'apprentissage en général, et de l'acquisition d'une langue en particulier, déplace la focalisation d'une analyse éducative des capacités intellectuelles et des environnements des étudiants en tant qu'entités fixes, vers les processus et les réponses dynamiques que ces derniers formulent et initient personnellement afin de parvenir à des buts qui comptent pour eux. Selon la théorie sociocognitive de Bandura (2009), aucun mécanisme de motivation personnelle n'est plus décisif que la croyance qu'ont les individus en leur capacité à exercer de l'influence sur ce qu'ils font et deviennent : «Si d'autres facteurs peuvent servir de guides ou de motivateurs, ils n'en sont pas moins enracinés dans la croyance centrale selon laquelle on possède la capacité de produire des effets par ses actions.» (Bandura, 2009, p. 28) Tout apprenant en langues a besoin de croire que, s'il le veut, il est capable de parvenir à communiquer efficacement dans la nouvelle langue qu'il étudie.

Dans le domaine de la recherche sur la motivation à apprendre une langue étrangère, Ushioda (2008) suggère que si l'élève ou l'étudiant qui apprend une langue ne se reconnait pas comme «agent» dans le contrôle qu'il parvient à exercer sur sa motivation, son mode de pensée et d'évaluation personnel concernant ses aptitudes en tant qu'apprenant risque de devenir négatif. Ses croyances personnelles - souvent implicites - jouent un rôle déterminant par rapport à la façon dont elles l'aident ou le freinent, souvent à son insu, dans l'exercice de son «agentivité» dans des contextes tant scolaires que sociaux. Croire, par exemple, qu'il faille être doué ou avoir un talent particulier pour apprendre une langue, ou qu'il soit nécessaire de vivre à l'étranger pour progresser dans une langue, sont des idées qui peuvent miner la persévérance et la confiance en soi dont l'élève, l'étudiant ou l'adulte en formation ont besoin pour profiter pleinement de leurs apprentissages en salle de classe (Ryan \& Mercer, 2011).

Pour Bandura (2009), l'agentivité est une capacité certes individuelle, mais elle émerge dans le contexte des interactions que chacun a avec autrui. La capacité agentique humaine s'enracine dans notre participation, en tant qu'êtres biologiques, à des pratiques socioculturelles. Or, une fois qu'elle émerge, elle peut jouer un rôle causal unique 
dans le développement subséquent du soi et de la société (Martin, 2004, p. 139; notre traduction). Les activités cérébrales sous-tendant la capacité agentique des individus ne sont pas ontologiquement réductibles, précisément parce que de tels processus mentaux nécessitent aussi une interaction au sein d'un monde social extérieur à l'organisme. L'agentivité est (et ne cesse de devenir) une propriété psychologique créée par le truchement de processus cérébraux complexes. Ceux-ci sont initiés et soutenus dans et par le contexte social pour produire à terme un niveau d'être ontologique qui se distingue, d'une manière nouvelle, des éléments biophysiques et socioculturels ayant présidé à sa création (ibid., p. 138).

L'agentivité est avant tout un processus dynamique qui se déploie à chaque instant de la vie. Même lors de décisions qui se prennent en un éclair, la perspective agentique met l'accent sur le fait qu'à tout instant quasiment de son existence (hors menace vitale), l'être humain opère des choix qui ont des conséquences pour lui-même et pour ce qui l'entoure (les autres comme son environnement). À priori donc, l'homme agentique mène une vie au cours de laquelle il s'efforce, tant bien que mal, de contrôler des évènements qui l'affectent. Il se sert de ses capacités d'autorégulation et d'autocontrôle pour tenter de concrétiser un avenir qu'il souhaite et prévenir un avenir qu'il ne désire pas (Bandura, 2009). Cependant, l'agentivité est loin d'être une «pure bénédiction». $\mathrm{Si}$ «mauvais usage » en est fait, l'aptitude à l'auto-influence et à l'autopersuasion peut également provoquer des dysfonctionnements et causer des souffrances (Bandura, 1999). Le phénomène de «défaillance du contrôle de soi» (self-regulation failure) en est un exemple saisissant (Baumeister, Heatherton \& Tice, 1994). L'incapacité croissante des individus à exercer un meilleur contrôle sur leurs propres conduites est en passe de devenir la toute première forme de pathologie sociale aux États-Unis. Le milieu éducatif, là-bas comme ici, est directement affecté par le phénomène (Charlot, 1997; Beaupère \& Boudesseul, 2009), et ce, dans un contexte où il devient de plus en plus difficile d'enseigner et où les demandes de la société en termes d'acquisition de compétences, notamment en langues, se font pressantes.

\section{Conclusion}

«Nos émotions occupent une place non négligeable dans notre quotidien. Elles ponctuent presque tous les moments significatifs de notre vie.» (Govaerts \& Grégoire, 2006, p. 97) Également reconnue désor- 
mais comme une variable incontournable dans le processus d'apprentissage (Mayer, Salovey \& Caruso, 2004), l'émotion constitue un facteur important au cœur de la réflexion didactique contemporaine. Selon l'approche sociocognitive adoptée ici (Bandura, 2003, 2009), l'émotion n'est pas vue comme un effet d'incitations situationnelles agissant directement sur les performances cognitives des élèves, mais plutôt comme un déterminant important de leur fonctionnement psychosocial dans son ensemble dont l'impact sur les comportements d'apprentissage s'opère au travers de mécanismes autorégulateurs.

Parallèlement, nous partons du principe qu'il est impossible d'envisager une amélioration des standards éducatifs sans soulever la question de la qualité de l'engagement et de la persistance des élèves dans l'effort. «L'efficacité de l'enseignement est dépendante de l'activité de l'élève puisque la modification de la structure cognitive exige un acte volontaire, un effort, une activité soutenue.» (Saint-Onge, 1991, p. 12) De même, Horwitz (1999) précise que puisque, à terme, l'apprentissage d'une langue passe inévitablement sous le contrôle de l'élève grandissant, il est impératif que la raison didactique conçoive des curricula qui tiennent compte de cette réalité sous-jacente. À l'âge de la centration sur l'acte d'apprendre et sur le développement de l'autonomie des élèves (Dam, 1995 ; Little, 2007), Ushioda (1996), comme Puozzo Capron (2009), évoque la nécessité d'une évolution importante dans le cahier des charges des enseignants. Au lieu de se poser la question de savoir comment il faut faire pour motiver leurs élèves, qu'est-ce que cela impliquerait s'ils transformaient la question pour s'intéresser davantage aux manières dont ils pourraient aider leurs élèves à (apprendre à) se motiver eux-mêmes (Ushioda, 1996, p. 2)?

«Apprendre c'est prendre des risques, affronter l'inconnu, risquer l'échec, la perte de l'estime d'autrui, et la sienne propre.» (André, 2006, p. 68) Si l'enseignant doit toujours maitriser la conception et la mise en œuvre de sa séquence didactique, ce qu'il doit pouvoir ajouter à cette compétence de base est la capacité d'amener l'élève «à se percevoir compétent $^{6}{ }$ (Puozzo Capron, 2009, p. 24) et de l'impliquer «subjectivement» dans son apprentissage et son utilisation de la langue cible dès les premiers cycles de l'enseignement (Ushioda, 1996, p. 64). La notion d'«instruction stratégique» développée par Oxford $(1990,2012)$

6. Pas seulement en termes de résultats, mais aussi, et peut-être surtout, en termes du processus d'apprentissage. 
offre aux enseignants des pistes d'intervention pour aider leurs élèves à devenir des apprenants plus efficaces et stratégiques, mieux autorégulés, et plus capables d'augmenter, si tel est leur choix et par leurs propres efforts, leurs chances de réussite dans la discipline.

Revenant au proverbe rûmî de départ, il est clair que l'enseignement du poète n'est pas aussi évident qu'il n'y parait. L'homme intelligent ne fait pas du contrôle de soi une obsession, pas plus que l'enfant ne s'intéresse qu'aux bonbons. L' «art de l'autocontrôle» dépend, entre autres, de la capacité - sociocognitivement acquise - à trouver une façon à la fois sensible et efficace de mettre à contribution, et non combattre, l'émotion. Il dépend d'une façon pour chacun d'apprendre à «porter sa biologie tout en étant porté par elle ${ }^{7}$ » d'une façon pour nos écoles, universités et communautés éducatives de parvenir à une meilleure maitrise de leur agentivité collective, notamment dans la dimension socio-affective de celle-ci, tout en restant «écolières d'elles-mêmes ${ }^{8}$ ». À notre sens, c'est dans la quête patiente de cet «art» que doivent s'élaborer, jour après jour, l'apprentissage et l'enseignement de toute langue et de toute discipline.

\section{RÉFÉRENCES BIBLIOGRAPHIQUES}

Allwright, D. \& Balley, K. (1991). Focus on the Language Classroom. Cambridge : Cambridge University Press.

ANDRÉ, J. (2006). Éduquer à la motivation, cette force qui fait réussir. Paris : Harmattan.

Bandura, A. (1999). Social Cognitive Theory of Personality. Dans L. Pervin \& O. John (dir.), Handbook of Personality (2e éd., p. 154-196). New York : Guilford.

B ANDURA, A. (2003). Auto-efficacité. Le sentiment d'efficacité personnelle (J. Lecomte, trad.). Paris, Bruxelles : De Boeck. (Ouvrage original publié en 1997 sous le titre Self-Efficacy: The Exercise of Control. New York : W. H. Freeman \& Co).

7. En référence à l'expression de Zundel (1996, dans Trocmé-Fabre, 1999, p. 37). Si Zundel (1996) suggère que l'Homme apprenne à «porter sa biologie au lieu d'être porté par elle», l'objet ici est d'essayer de concilier les deux points de vue en un seul processus de devenir.

8. Selon les termes de Casanova (dans Trocmé-Fabre, 1999, p. 37). 
BANDURA, A. (2009). La théorie sociale cognitive : une perspective agentique (S. Brewer \& P. Carré, trad.). Dans P. Carré \& F. Fenouillet (dir.), Traité de psychologie de la motivation (p. 15-45). Paris : Dunod. (Chapitre original publié en 2001).

Baumeister, R. F., Heatherton, T. F. \& Tice, D. M. (1994). Losing Control: How and Why People Fail at Self-Regulation. San Diego : Academic Press.

Beaupère, N. \& Boudesseul, G. (dir.). (2009). Sortir sans diplôme de l'Université : comprendre les parcours d'étudiants «décrocheurs». Paris : La documentation française.

Berglas, S. \& Baumeister, R. F. (1993). Your Own Worst Enemy: Understanding the Paradox of Self-Defeating Behavior. New York : Basic Books.

Bouffard, T. (2011). Des apprenants autonomes? Dans É. Bourgeois \& G. Chapelle (dir.), Apprendre et faire apprendre (p. 139-151). Paris : PUF.

Bourgeols, É. (2011). La motivation à apprendre. Dans É. Bourgeois \& G. Chapelle (dir.), Apprendre et faire apprendre (p. 235-253). Paris : PUF.

Brewer, S. S. (2010). Un regard agentique sur l'anxiété langagière. Dans J. Aden, T. Grimshaw \& H. Penz (dir.), Enseigner les langues-cultures à l'ère de la complexité : approches interdisciplinaires pour un monde en reliance (p. 75-88). Bruxelles : Peter Lang.

Brown, H. D. (2007). Principles of Language Learning and Teaching ( $5^{\mathrm{e}}$ éd.). White Plains, NY : Pearson Longman.

CArré, P. (2004). Bandura : une psychologie pour le XXI e siècle. Savoirs, hors série, Autour de l'œuvre d'Albert Bandura (p. 9-50). Paris : Harmattan.

CARRÉ, P. (2005). L'Apprenance : vers un nouveau rapport au savoir. Paris: Dunod.

Chambers, G. N. (1999). Motivating Language Learners. Clevedon : Multilingual Matters.

Charlot, B. (1997). Du Rapport au Savoir. Éléments pour une théorie. Paris : Anthropos.

COMMISSION EUROPÉENnE (1995). Enseigner et apprendre - Vers la société cognitive. Luxembourg : Office des publications officielles des communautés européennes.

Cosnefroy, L. (2011). L'apprentissage autorégulé, entre cognition et motivation. Grenoble : PUG.

DAM, L. (1995). Learner Autonomy 3: From Theory to Classroom Practice.

Dublin : Authentik. 
Damasio, A. R. (1995). L'erreur de Descartes. La raison des émotions. Paris : Odile Jacob.

Deci, E. L. \& Ryan, R. M. (1991). A motivational Approach to Self: Integration in Personality. Dans R. A. Dienstbier (dir.), Perspectives on Motivation (p. 237-288). Nebraska Symposium on Motivation, 1990, vol. 38. Lincoln : University of Nebraska Press.

Eccles, J. S. \& Midgley, C. (1989). State-Environment Fit: Developmentally Appropriate Classrooms for Young Adolescents. Dans R. Ames \& C. Ames (dir.), Research on Motivation in Education. Vol. 3, Goals and cognitions (p. 139-186). New York : Academic Press.

Fenouillet, F. (2009). Vers une intégration des conceptions théoriques de la motivation. Note de synthèse pour l'habilitation à diriger des recherches. Université Paris-Ouest La Défense.

Ford, M. E. (1992). Motivating Humans: Goals, Emotions, and Personal Agency Beliefs. Newbury Park : Sage.

FrankL, V. (1962). Man's Search for Meaning: An introduction to Logotherapy. Boston : Beacon Press.

Fridda, N. H. (1988). The Laws of Emotion. American Psychologist, 43, 349-358.

Govaerts, S. \& GréGoire, J. (2006). Motivation et émotions dans l'apprentissage scolaire. Dans B. Galand \& É. Bourgeois (dir.), (Se) Motiver à apprendre (p. 97-106). Paris : PUF.

Guiora, A. Z. (1983). The Dialectic of Language Acquisition. Language Learning, 33, 3-12.

Horwitz, E. K. (1999). Preface. Dans D. J. Young (dir.), Affect in Foreign Language and Second Language Learning: A Practical Guide to Creating a Low-Anxiety Classroom Atmosphere (p. XI-XII). Boston : McGraw-Hill College.

Horwitz, E. K., Horwitz, M. B. \& Cope, J. (1986). Foreign Language Classroom Anxiety. The Modern Language Journal, 70, 125-132.

JANET, P. (1928). De l'angoisse à l'extase. Paris : Alcan.

Kitayama, S. \& Howard, S. (1994). Affective Regulation of Perception and Comprehension: Amplification and Semantic Priming. Dans P. M. Niederthal \& S. Kitayama (dir.), The Heart's Eye: Emotional Influences in Perception and Attention (p. 41-65). San Diego, CA : Academic Press.

LeDoux, J. (1996). The Emotional Brain: The Mysterious Underpinnings of Emotional Life. New York : Touchstone.

LitTle, D. (2007). Language Learner Autonomy: Some Fundamental Considerations Revisited. Innovations in Language Learning and Teaching, 1 (1), 14-29. 
Little, D., Ridley, J. \& Ushioda, E. (2002). Towards Greater Learner Autonomy in the Foreign Language Classroom. Dublin : Authentik.

Luminet, O. (2002). Psychologie des émotions. Confrontation et évitement. Bruxelles : De Boeck.

McGonigal, K. (2012). The Willpower Instinct: How Self-Control Works, Why It Matters, and What You Can Do to Get More of It. New York : Avery (Penguin Group).

MacIntyre, P. D. (1995). How Does Anxiety Affect Second Language Learning? A Reply to Sparks and Ganschow. Modern Language Journal, 79, 1-32.

Martin, J. (2004). Self-Regulated Learning, Social Cognitive Theory, and Agency. Educational Psychologist, 39 (2), 135-145.

Maslow, A. H. (1968). Toward a Psychology of Being (2 éd., p. 179-185). New York : Van Nostrand Reinhold.

Mayer, J. D. \& Salovey, P. (1995). The Intelligence of Emotional Intelligence. Intelligence, 17, 433-442.

Mayer, J. D., Salovey, P. \& Caruso, D. R. (2004). Emotional Intelligence: Theory, Findings, and Implications. Psychological Inquiry, 15, $197-$ 215.

MeIrIeu, P. (1987). Apprendre... oui, mais comment? Paris : ESF.

Musch, J. \& Klauer, K. C. (2003). The Psychology of Evaluation. Mahwah : Erlbaum.

OXFord, R. (1990). Language Learning Strategies: What Every Teacher Should Know. Rowley, MA : Newbury House.

OXFORD, R. (2012). Teaching and Researching Language Learning Strategies. Harlow, UK : Pearson Longman.

Piaget, J. (1971). Biology and Knowledge. Chicago : University of Chicago Press.

Posner, M. I. \& Snyder, C. R. R. (1975). Attention and Cognitive Control. Dans R. L. Solso (dir.), Information Processing and Cognition: The Loyola Symposium. Mahwah, NJ : Lawrence Erlbaum.

Puozzo Capron, I. (2009). Le sentiment d'efficacité personnelle. Pour un nouvel enseignement/apprentissage des langues. Revue Sciences Croisées, 6, 1-29. Disponible sur <http://sciences-croisees.com/N6/Puozzo Capron.pdf>.

Ridley, D. S. (1991). Reflective Self-Awareness: A Basic Motivational Process. Journal of Experimental Education, 60 (1), 31-48.

Roeser, R. W. \& Peck, S. C. (2009). An Education in Awareness: Self, Motivation, and Self-Regulated Learning in Contemplative Perspective. Educational Psychologist, 44 (2), 119-136.

Ryan, S. \& Mercer, S. (2011). Natural Talent, Natural Acquisition and Abroad: Learner Attributions of Agency in Language Learning. Dans 
G. Murray, X. Gao \& T. Lamb (dir.), Identity, Motivation and Autonomy in Language Learning (p. 160-176). Bristol, UK : Multilingual Matters. Saint-Onge, M. (1991). L'étude : quel problème? Pédagogie collégiale, 5 (1), 12-16. Disponible sur <www.cvm.qc.ca/aqpc/Auteurs/Saint-Onge, $\% 20 \mathrm{Michel} /$ Saint-Onge, \%20Michel\%20(05,1).pdf $>$.

SAPOlsky, R. M. (2004). The Frontal Cortex and the Criminal Justice System. Philosophical Transactions of the Royal Society of London, Series B, Biological Sciences, 359, 1787-1796.

Schumann, J. H. (1997). The Neurobiology of Affect in Language. Oxford : Blackwell.

Schunk, D. (1990). Goal Setting and Self-Efficacy During Self-Regulated Learning. Educational Psychologist, 25, 51-69.

Syssau, A. (2006). Émotion et cognition. Dans N. Blanc (dir.), Émotion et cognition. Quand l'émotion parle à la cognition. Paris : éditions In Press.

Trocmé-Fabre, H. (1999). Réinventer le métier d'apprendre. Paris : Éditions d'Organisation.

Ushioda, E. (1996). Learner Autonomy 5 : The Role of Motivation. Dublin : Authentik.

Ushioda, E. (2008). Motivation and Good Language Learners. In C. Griffiths (dir.), Lessons from Good Language Learners (p. 19-34). Cambridge : CUP.

Wittezaele, J.-J. (2003). L'homme relationnel. Paris : Seuil.

Wolters, C. (1998). Self-Regulated Learning and College Students' Regulation of Motivation. Journal of Educational Psychology, 90, 224-235.

Zajonc, R. B. (1984). On the Primacy of Affect. American Psychologist, 39, 117-123.

Zimmerman, B. Z. (1990). Self-Regulated Learning and Academic Achievement: An Overview. Educational psychologist, 25, 3-17.

Zundel, M. (1996). À l'écoute du silence. Paris : Éd. Tequi. 